\title{
Drug resistant enterococci: factors associated with gastrointestinal tract colonization
}

\author{
S. P. Gunasekera', ${ }^{*}$. Perera ${ }^{2}$ \\ The Ceylon Journal of Medical Science 2007; 50: 9-14
}

\begin{abstract}
Resistant enterococci are recognized as important hospital acquired pathogens. They are generally sensitive to ampicillin but intrinsically resistant to cephalosporins and aminoglycosides and are known to acquire high level resistance to these drugs. High level resistance has serious implications for treatment of patients infected with these organisms and for infection control activities. Colonisation of the gastrointestinal tract is a prerequisite to infection. A number of factors are known to contribute to acquiring colonisation by resistant enterococci according to studies conducted in the West. The present study determines the prevalence of resistant enterococci on admission in a cohort of hospitalised patients, and identifies some risk factors that are significantly associated with acquiring resistant enterococci during hospital stay.
\end{abstract}

A prospective study conducted over three months, on 125 patients in orthopaedic units and the burns unit of the National Hospital of Sri Lanka, to determine colonisation showed that $5.6 \%$ were colonised on admission and $16.8 \%$ acquired ampicillin resistant enterococci (ARE) during hospitalisation. No vancomycin resistant enterococci (VRE) were isolated. Risk factors that were significantly associated with colonisation were empiric use of antibiotics, treatment with ampicillin, multiple use of antibiotics, prior hospitalisation and presence of diabetes mellitus. These features will be helpful in identifying possible patients for screening for resistant enterococci, with a view to implementing more stringent infection control measures to prevent spread of these organisms.

Key words: Colonization, enterococci, orthopaedic units, burns unit.

\section{Introduction}

Enterococci are robust, facultative anaerobic bacteria and form part of the normal intestinal flora of humans and animals. They are present as regional flora of the bowels of over $90 \%$ of healthy humans. They began to be recognized as a common cause of hospital associated infections in the 1970s. Enterococci do not possess the same intrinsic virulence of certain other Gram positive organisms, such as Streptococcus pyogenes or Staphylococcus aureus, but are capable of causing serious infections including bacteraemia, endocarditis and meningitis. The major reason related to this fact is their intrinsic resistance to a number of antibiotics and their ability to acquire new resistance determinants (1).

\section{Senior Registrar in Microbiology}

2. Professor, Department of Microbiology, Faculty of Medicine, University of Colombo, Sri Lanka.

* Author for correspondence. Department of Microbiology, Faculty of Medicine, Kynsey Road, Colombo 8, Sri Lanka. 
Enterococci are generally sensitive to ampicillin but are intrinsically resistant to many antibiotics including cephalosporins, cotrimoxazole, clindamycin and aminoglycosides. However, when a beta lactam drug or vancomycin is combined with an aminoglycoside, the intrinsic resistance can be overcome due to a synergistic effect. They can acquire high level resistance to aminoglycosides, penicillin group of antibiotics and vancomycin and then this synergistic killing is absent in these strains. The acquisition of vancomycin resistance has serious implications in management of infections as they are frequently resistant to all antibiotics that are effective in the treatment of vancomycin susceptible enterococci (2).

It is recognised that colonization of the gastrointestinal tract with vancomycin resistant enterococci (VRE) plays a major role in the development of subsequent infections. During surveys it has been found that 26 to $41 \%$ of health care workers carried VRE on their hands (3). Resistant enterococci also have been cultured from medical equipments and devices (4). Thus faecal VRE are important as sources of infection and also for hospital associated spread.

Antibiotic use, especially empiric and broad spectrum regimens, exposure to vancomycin and enteral tubal feeding are reported as important factors leading to colonization with resistant enterococci. Other risk factors include serious underlying disease, length of hospital stay, prior surgery, renal insufficiency, presence of urinary or vascular catheters and treatment in intensive care units (5).

This study was carried out to examine the prevalence of rectal colonization with both ampicillin and vancomycin resistant enterococci at the time of admission and during the hospital stay in patients admitted to orthopaedic units and the burns unit and to determine risk factors for such colonization. These units were selected as patients spend a longer duration in them compared to other wards and as use of antibiotics is comparatively higher in them.

\section{Design, setting and methods}

A prospective study was carried out over three months on 125 patients admitted to a 559 bed orthopaedic department consisting of three units and a 57 bed burns unit at the National Hospital of Sri Lanka. One hundred and twenty five patients admitted on weekdays were selected for the study where their hospital stay was expected to be $>2$ weeks. Although a sample size of 125 was not the best for a study of this nature, only 125 patients were recruited as the study had to be completed in 3 months. Ethical clearance for the study was obtained from the Ethical Review Committee of the Faculty of Medicine, Colombo. Three rectal swabs were obtained from each patient, first on admission or transfer to the study units and repeated at weekly intervals for two weeks.

The swabs were cultured on two plates of Enterococcosal agar (Difco) each containing $6 \mu \mathrm{g} / \mathrm{ml}$ ampicillin and $16 \mu \mathrm{g} / \mathrm{ml}$ vancomycin. After incubation for 48 hours, positive cultures which hydrolysed aesculin bile were identified as enterococci by colony morphology, gram stain and a negative catalase test. The identification was confirmed with group D latex reagent (Murex Diagnostics) and by PYR test (Murex Diagnostics) which shows hydrolysis of Lpyrrolidonyl -B naphthylamide due to the presence of pyrrolidonyl arylamidase in the organisms $(6,7)$. Susceptibility testing to ampicillin and vancomycin was performed by the NCCLS method (8). Beta lactamase production was detected by the acidometric method (9).

Demographic data and information on 
previous hospitalization, antibiotic use and presence of co-morbid factors such as diabetes, malignancy etc was obtained from all patients. The treatment charts were reviewed daily to obtain data on antimicrobial therapy. The Fisher's exact test and chi square $\left(\mathrm{X}^{2}\right)$ test were used to assess the statistical significance of data analysed using the Epi info version 6 statistical package.

\section{Results}

There were 69 males and 56 females. The mean age of the study group was 51.9 years $(\mathrm{SD}=24.1 \mathrm{y})$ and the range was 6 to 98 years. Mean duration of hospitalization was $17.2 \pm 4.3$ days with a range of 3 to 29 days. Two patients were excluded from the study as they were discharged before any repeat swabs were obtained. Seventy three patients had been admitted from the community and 52 had been transferred; 20 from within the hospital and the balance 32 from 15 different hospitals. Sixteen patients were diabetic.

A total of $28(22.4 \%)$ patients were colonized with ampicillin resistant enterococci (ARE) as confirmed by antibiotic sensitivity testing. Among these, 7 were carrying ARE on admission to the study units and 21 became positive for ARE during the hospital stay.
Further analysis of the 7 patients who were colonised showed that 6 of them were transferred from another ward of the same hospital or from another hospital. Only one patient was admitted directly from the community. None of the patients had VRE. There were no beta lactamase producing strains.

The comparison of the 7 patients who had ARE on admission with those who did not have ARE on admission showed that transfer from another ward or hospital was a significant risk factor for colonization $\left(X^{2}=5.94 ; p, 0.020\right.$, Table 1$)$.

On comparison of the 21 patients who acquired ARE after admission with those who never became colonized, presence of diabetes $(33.3 \%$ versus $8.4 \%, \mathrm{p}, 0.002)$ and antibiotic therapy $(80.9 \%$ versus $45.3 \%$, $\mathrm{p}, 0.003)$ were found to be significantly associated with colonization (Table 2). Antibiotics were used either empirically (treatment of a presumed infection for which no specific aetiological agent has been determined) or prophylactically (prior to surgery) or therapeutically (used when microbiologically confirmed or clinically documented infection is present) among the study population. Further analysis of antibiotic therapy showed that empiric use

Table 1. Risk factors for colonisation with ampicillin resistant enterococci (ARE) on admission

\begin{tabular}{llc}
\hline Risk factor & \multicolumn{2}{l}{ Colonisation status $(\mathrm{n}=125)$} \\
& Positive & Negative \\
\hline Admitted from the community & 1 & 72 \\
Transfer from another ward or hospital & 6 & 46 \\
\hline Total & 7 & 118 \\
\hline
\end{tabular}


(52.3\% versus $18.9 \%, \mathrm{P}<0.001)$ was a significant risk factor for colonization with ARE. However, therapeutic $(9.5 \%$ versus $2.1 \%, \mathrm{p}, 0.092)$ or prophylactic use $(19 \%$ versus $24.2 \%, \mathrm{p}, 0.612$ ) of antibiotics were not significant risk factors. Use of more than 2 antibiotics was a significant risk factor for acquisition of ARE (28.6\% versus $6.3 \%$ ), $\mathrm{p}, 0.002)$. Cephalosporins were used minimally on the study group. Because of that, the effect of cephalosporin use on ARE colonization was not determined in this study. However, ampicillin or amoxicillin was used on $8(38.1 \%)$ of the 21 patients who were colonised with ARE compared to $7(7.4 \%)$ of the 95 patients who were not colonised. The difference was statistically significant $(p<0.001)$. Similarly, hospitalisation was found to be significantly associated with acquisition of ARE in this study $\left(X^{2}=15.59 ; P<0.01\right.$, Table 3$)$. However, there was no significant difference between colonisation at 7 days and 14 days of hospitalisation. The significance of other comorbid factors such as malignancy, abnormal mental status, diarrhoea, bladder and bowel incontinence which were recognized as significant risk factors in previous studies could not be studied due to the small number of patients with such risk factors (Table 2).

\section{Discussion}

Studies conducted worldwide have documented an alarming increase in hospital acquired infections due to resistant enterococci (10). These infections require treatment with expensive and potentially toxic drugs and carry a high morbidity. Gastrointestinal carriage of resistant enterococci is a key factor determining development of infection by the same organism (11). Furthermore, colonising flora provides an important clinically hidden reservoir of resistant enterococcal strains for subsequent hospital acquired infection and transmission. If factors promoting colonization are identified and controlled, it may be possible to decrease the incidence of colonisation and thereby clinical infection.

The present study was designed to ascertain prospectively the rates and risk factors for hospital acquired rectal colonisation with resistant enterococci in orthopaedic units and the burns unit in a tertiary care hospital. The importance of gastrointestinal colonisation of resistant enterococci is reflected in the results. About a quarter $(22.4 \%)$ of the patients harboured ampicillin resistant enterococci at some point of their hospital stay. None were colonised with vancomycin resistant enterococci.

Not surprisingly, hospitalization was a significant risk factor for colonisation with resistant enterococci as 6 of the 7 patients who were colonized on admission had been transferred from another unit or hospital (Table 1).

Similar to findings in previously published studies, antimicrobial therapy was significantly associated with nosocomial colonisation with ARE. According to Weinstein's study $33(9.4 \%)$ patients were colonized during hospital stay and independent risk factors for hospital acquisition of ARE included, treatment with more than three antibiotics and the use of third generation cephalosporins (9). In the present study treatment with multiple antibiotics was a significant risk factor. Empiric antimicrobial therapy was a more significant risk factor compared to therapeutic and prophylactic antimicrobial therapy. Ampicillin use was a highly significant risk factor for the development of ARE. The presence of diabetes was also a significant risk factor. However, it is important to note that diabetic patients receive antibiotics more often and for a longer duration than non diabetics. 
Table 2. Risk factors for colonisation in patients with hospital associated acquisition of ampicillin resistant enterococci (ARE)

\begin{tabular}{|c|c|c|c|c|}
\hline \multirow[t]{2}{*}{ Risk factor } & \multicolumn{2}{|c|}{ Colonisation status } & \multirow[b]{2}{*}{$\begin{array}{r}\text { Odds ratio } \\
\text { in } 95 \% \text { CI }\end{array}$} & \multirow[b]{2}{*}{$\mathrm{p}$ value } \\
\hline & $\begin{array}{c}\text { Positive }(\mathrm{n}=21) \\
\text { No. }(\%)\end{array}$ & $\begin{array}{c}\text { Negative }(n=95) \\
\text { No. }(\%)\end{array}$ & & \\
\hline Diabetes & $7(33.3)$ & $8 \quad(8.4)$ & 5.44 & 0.002 \\
\hline Malignancy & $1 \quad(4.8)$ & $3(3.1)$ & 1.53 & 0.715 \\
\hline Abnormal mental status & $1 \quad(4.8)$ & $6(6.3)$ & 0.74 & 0.767 \\
\hline Bladder bowel incontinence & $5(23.8)$ & $13(13.7)$ & 1.47 & 0.246 \\
\hline Diarrhoea & $2 \quad(9.5)$ & $4 \quad(4.2)$ & 2.39 & 0.320 \\
\hline Antibiotic use & $17(80.9)$ & $43(45.3)$ & 8.78 & 0.003 \\
\hline Empiric & $11(52.3)$ & $18(18.9)$ & 4.71 & $<0.001$ \\
\hline Therapeutic & $2 \quad(9.5)$ & $2(2.1)$ & 4.89 & 0.092 \\
\hline Prophylactic & $4(19.0)$ & $23(24.2)$ & 0.74 & 0.612 \\
\hline Use of $>2$ antibiotics & $6(28.6)$ & $6(6.3)$ & 5.93 & 0.002 \\
\hline Use of cephalosporins & $0 \quad(0)$ & $5 \quad(5.3)$ & 0 & 0.282 \\
\hline Use of ampi/amoxy & $8(38.1)$ & $7 \quad(7.4)$ & 7.74 & $<0.001$ \\
\hline
\end{tabular}

Table 3. Hospitalisation as a risk factor for nosocomial acquisition of ampicillin resistant enterococci $(\mathrm{ARE}) \mathrm{n}=123$

\begin{tabular}{cc}
\hline Day of admission & ARE positive \\
\hline 0 & 7 \\
7 & 15 \\
14 & 28 \\
\hline
\end{tabular}

Chi-square 15.59; $\mathrm{p}<0.01$

In conclusion, this study helps to elucidate some epidemiological factors of ARE in a cohort of hospitalised patients. Routine screening of all patients for resistant enterococci on admission is impractical due to financial constraints. Screening the high risk groups and instituting antibiotic restriction programmes may limit introduction and spread of resistant strains in the units.

\section{Limitations of the study}

This study was confined to patients in orthopaedic units and the burns unit only due to limited funds allocated for the study. Conclusion about the risk factors associated with ARE colonisation have been drawn using a subgroup of 28 patients.

\section{Acknowledgements}

We thank Doctors Upali Banagala, Vasantha Perera, Narendra Pinto, G L Punchihewa, the consultant orthopaedic surgeons, Dr Chandani Perera, consultant plastic surgeon, all staff of these units at the National Hospital of Sri Lanka for their assistance and staff of the Department of Microbiology, Faculty of Medicine, Colombo. 
Mr. Sanath Mahawithanage for assistance in statistical analysis of data, and the patients for participating in the study.

\section{References}

1. Moellering R.C. The enterococcus: a classic example of the impact of antimicrobial resistance on therapeutic options. Journal of Antimicrobial Chemotherapy 1991; 28; 1-12.

2. Murray B.E. The life and times of the enterococcus. Clinical Microbiology Reviews 1990; 3: 46-59.

3. Jarvis W.R. The epidemiology of colonisation. Infection Control and Hospital Epidemiology 1996; 17: 47-52.

4. Donskey C.J., Salata R.A. Enterococcal infections,Omaha, Nebraska, USA. (http//www.emedicine<com/med/ topic680.htm. Accessed 20th May 2006.)

5. Mandell G.L., Bennett J.E. and Dollin R. Mandell, Douglas and Benett's Principles and practice of infectious diseases, 4th Edition, Volume 2, Philadelphia: Churchill Livingstone. 1995: p1826-1834.

6. Murray P.R., Baron E.J., Pfaller M.A., Tenover F.C., Yolken R.H. Manual of Clinical Microbiology, 6th Edition,
Washington, American Society of Microbiology (ASM) Press, pp308-315, 1327-1341, 1356-1367.

7. Barrow G.I., Feltham R.K.A. Cowan and Steels manual for the identification of medical bacteria, Third edition, Cambridge University Press, 1993; p225-226.

8. National Committee for Clinical Laboratory Standards (NCCLS). Performance standards for antimicrobial susceptibility testing, Twelfth international supplement, 2002; volume 22, No 1: 48-51.

9. Dyck E.V., Meheus A.Z., Piot P. Laboratory diagnosis of sexually transmitted diseases, World Health Organisation (WHO) 1999; p11.

10. Weinstein J.W., Roe M., Towns M., Sanders L., Thorpe J.J., Corey G.R. and Sexton D.J. Resistant enterococci : A prospective study of prevalence, incidence and factors associated with colonisation in a University hospital. Infection Control and Hospital Epidemiology 1996; 17 : 36-41.

11. French G.L. Enterococci and vacomycin resistance. Clinical Infectious Disease 1998; 27: Suppl 1; S75-83. 